\title{
Neutralizing antibodies against coronaviruses
}

Pamela Bjorkman

California Institute of Technology, Pasadena, California, United States

We are using single-particle cryo-electron microscopy (cryo-EM) to solve structures of infection- and vaccination-induced antibodies complexed with the spike trimer of SARS-CoV-2 in order to elucidate the structural correlates of antibody-based immune protection. Structural comparisons allowed us to classify antibodies against the receptor-binding domain (RBD) of spike trimer into categories: (1) neutralizing antibodies encoded by the VH3-53 gene segment with short CDRH3 loops that block the host receptor ACE2 and bind only to 'up' RBDs; (2) ACE2-blocking neutralizing antibodies that bind both up and 'down' RBDs and can contact adjacent RBDs; (3) neutralizing antibodies that bind outside the ACE2 site and recognize both up and down RBDs; and (4) previously described antibodies that do not block ACE2 and bind only to up RBDs. Class 2 contained four neutralizing antibodies with epitopes that bridged RBDs, including a VH3-53 antibody that used a long CDRH3 with a hydrophobic tip to bridge between adjacent down RBDs, thereby locking the spike into a closed conformation. Epitope and paratope mapping revealed few interactions with host-derived $N$-glycans and minor contributions of antibody somatic hypermutations to epitope contacts. Affinity measurements and mapping of naturally occurring and in vitro-selected spike mutants in 3D provided insight into the potential for SARS-CoV-2 to escape from antibodies elicited during infection or delivered therapeutically. These classifications and structural analyses provide rules for assigning current and future human RBD-targeting antibodies into classes, evaluating avidity effects and suggesting combinations for clinical use, and provide insight into immune responses against SARS CoV-2. Our structural studies have also guided the development of a potential pan-betacoronavirus vaccine. The vaccine approach involves co-display of diverse sets of RBDs from SARS-like beta coronaviruses (sarbecoviruses) on nanoparticles (mosaic-RBD-nanoparticles) that results in increased breadth of neutralizing responses in mice compared with nanoparticles presenting only SARS-CoV-2 RBDs. An advantage of this approach is that we have shown that RBD-mi3 nanoparticles retain immunogenicity after lyophilization, suggesting they could be easily stored for widespread use when needed. Thus this modular vaccine platform could provide protection from SARS-CoV-2 as well as potential future emergent coronaviruses that could cause pandemics.

\section{References}

Barnes, CO, West Jr., AP, Huey-Tubman, KE, Hoffmann, MAG, Sharaf, NG, Hoffman, PR, Koranda, N, Gristick, HB, Gaebler, C, Muecksch, F, Cetrulo Lorenzi, JC, Finkin, S, Hägglöf, T, Hurley, A, Millard, KG, Weisblum, Y, Schmidt, F, Hatziioannou, T, Bieniasz, PD, Caskey, M, Robbiani, DF, Nussenzweig, MC, Bjorkman, PJ (2020) Structures of human antibodies bound to SARS-CoV-2 spike reveal common epitopes and recurrent features of antibodies, Cell 182: 828-842 PMCID: PMC7311918 doi:10.1016/j.cell.2020.06.025

Barnes, CO, Jette, CA, Abernathy, MA, Dam, K-M A, Esswein, SR, Gristick, HB, Malyutin, AG, Sharaf, NG, Huey-Tubman, KE, Lee, YE, Robbiani, DF, Nussenzweig, MC, West, AP, Bjorkman, PJ (2020) SARS-CoV-2 neutralizing antibody structures inform therapeutic strategies. Nature 588: 682-687 doi:10.1038/s41586-020-2852-1

Cohen, AA, Gnanapragasam, PNP, Lee, YE, Hoffman, PR, Ou, S, Kakutani, LM, Keeffe, JR, Wu, H-J, Howarth, M, West, AP, Barnes, CO, Nussenzweig, MC, Bjorkman, PJ (2021) Mosaic nanoparticles elicit 
cross-reactive immune responses to zoonotic coronaviruses in mice. Science eabf6840 doi: $10.1126 /$ science.abf6840 